\title{
Phosphodiesterase inhibitors as a new generation of antiprotozoan drugs: exploiting the benefit of enzymes that are highly conserved between host and parasite
}

\author{
Thomas Seebeck $^{\dagger, 1}$, Geert Jan Sterk ${ }^{2}$, and Hengming $\mathrm{Ke}^{3}$ \\ ${ }^{1}$ Institute of Cell Biology, University of Bern, Baltzerstrasse 4, CH-3012 Bern, Switzerland \\ ${ }^{2}$ Mercachem, Kerkenbos 1013, PO Box 6747, 6503 Ge Nijmegen, The Netherlands ${ }^{3}$ Department \\ of Biochemistry and Biophysics, University of North Carolina, Chapel Hill, NC 27599-7260, USA
}

\begin{abstract}
Protozoan infections remain a major unsolved medical problem in many parts of our world. A major obstacle to their treatment is the blatant lack of medication that is affordable, effective, safe and easy to administer. For some of these diseases, including human sleeping sickness, very few compounds are available, many of them old and all of them fraught with toxic side effects. We explore a new concept for developing new-generation antiprotozoan drugs that are based on phosphodiesterase (PDE) inhibitors. Such inhibitors are already used extensively in human pharmacology. Given the high degree of structural similarity between the human and the protozoan PDEs, the vast expertise available in the human field can now be applied to developing disease-specific PDE inhibitors as new antiprotozoan drugs.
\end{abstract}

\section{The medical problem}

Protozoan diseases have always been faithful companions of mankind and are still prominently with us today [1-3]. Nevertheless, their effect on human health and wellbeing is dramatically different depending on the region of the world considered. In the industrialized societies in temperate climes, a number of protozoan pathogens are common, but rarely cause serious disease, at least in immunologically competent individuals. Toxoplasmosis, caused by Toxoplasma gondii, is a widespread infection that rarely causes overt symptoms in otherwise healthy individuals. This relatively complacent picture can alter dramatically upon immunosuppression, or upon infection of a naive woman during pregnancy [4]. Here, the fetus can be seriously affected through transplacental infection, and it runs the additional risk of contracting a perinatally acquired ocular toxoplasmosis [5]. Trichomonas vaginalis is the most common nonviral, sexually transmitted disease agent worldwide. Since the infection is mostly asymptomatic, it often goes untreated and is a frequent cause of pregnancy-related complications or infertility. In addition, Trichomonas infections significantly increase the susceptibility of women for infection by HIV [6]. Several intestinal parasites can lead to unpleasant infections. These are usually self-limiting and rarely have serious consequences [7,8]. Among them, Giardia lamblia is the undisputed leader, with an estimated 280 million annual cases worldwide [9]. However, the constantly increasing number of immunocompromised individuals is cause for concern. While infections of immunologically competent individuals by intestinal parasites such as G. lamblia or

(C) 2011 Future Science Ltd

†Author for correspondence: Tel.: +41 318291 443, thomas.seebeck@izb.unibe.ch . 
Cryptosporidium hominis are usually self-limiting, they are frequently fatal in immunocompromised patients [1,10-12].

In stark contrast to the situation in industrialized countries, diseases caused by protozoan parasites constitute serious problems in many parts of the developing world. In terms of publicity, the clear leader is malaria, a complex of four diseases caused by the four protozoan species Plasmodium falciparum (the most important and most lethal), Plasmodium malariae, Plasmodium vivax and Plasmodium ovale. Very recently, yet another species, Plasmodium knowlesi, previously thought to cause malaria only in monkeys, was recognized as a fifth human malaria agent [13-15]. Approximately half of the world's population currently lives under the risk of malaria infections, corresponding to approximately 3.3 billion people in 109 countries. Only 35 of these countries (30 in Africa and five in Asia) contribute $98 \%$ of all malaria cases, and $89 \%$ of all of these occur in subSaharan Africa. In 2008 alone, an estimated 200 million malaria cases occurred, causing approximately one million deaths, mostly children below 5 years of age. Despite this high number of fatalities, the most severe impact of malaria is caused by its transient, but no less devastating, effects on human health and performance, resulting in an estimated annual loss of approximately 35 million disease-adjusted life years and US $\$ 12$ billion in direct losses to the economies of the afflicted countries $[16,17,201]$. Despite this devastation, malaria is only the tip of the iceberg of human suffering.

A broader view shows that, besides malaria, a large group of other protozoan infections also cause great misery and suffering in vast parts of the world. For many of these, good (according to modern standards of pharmacology) chemotherapy often does not exist. Among these other protozoan diseases, amoebiasis is probably the most widespread (approximately 10\% of people have their colon infected). Most of the infections are benign, with limited clinical manifestations. However, in a small percentage of cases, the amoeba emigrate from the colon and invade the liver. This leads to the formation of constantly growing liver abscesses that eventually take a fatal course if not treated in time [18]. While amoebiasis was traditionally ascribed to a single species, Entamoeba histolytica, it has now become clear that at least three morphologically indistinguishable species can infect the colon. Two of them, Entamoeba dispar and Entamoeba moshkovskii, are thought to cause the self-limiting, colon-restricted disease, while only E. histolytica has the capability of invasiveness. The quantitative role of these three species in amoebiasis epidemiology remains to be clarified [19].

Another important group of protozoan pathogens are the kinetoplastida, a vast group of highly motile unicellular organisms propelled by a single flagellum and characterized by a particular substructure of the mitochondrion, the kinetoplast. Most members of this order are pathogens of plants or animals, and several of them are of great medical and/or economical importance. The genus Phytomonas comprises several economically disastrous parasites of cash crops such as oil palm, coffee, coconut and tomato [20]. Various members of the genus Trypanosoma are major disease agents for domestic animals [21-23] and cause great economic losses in South America, Africa and South-East Asia, resulting from decreased meat and milk production, reduced indirect benefits, such as hides, manure or traction power, and the loss of highly priced breeding horses or racing camels.

In addition to these veterinary parasites, three members of the genus Trypanosoma, Trypanosoma brucei gambiense, Trypanosoma brucei rhodesiense and Trypanosoma cruzi, as well as several members of the genus Leishmania including Leishmania major, Leishmania donovani or Leishmania amazonensis, cause severe human diseases [24]. The African trypanosomes T.b. rhodesiense and T.b. gambiense cause human sleeping sickness in sub-Saharan Africa. They are transmitted by the blood-sucking tsetse fly between human 
hosts or between humans and animal reservoirs, such as domestic or game animals. The disease may take a protracted course and culminates in the infection of the central nervous system. It is inexorably fatal if not treated [25]. The South-American trypanosome T. cruzi is the causative agent of Chagas disease in much of southern and middle America, including the southern parts of the USA. The parasites are transmitted from animals to humans, or between humans by bedbugs that contaminate the bite wound with their parasite-containing feces during the blood meal. This disease is particularly insidious because, after an initial acute phase with a mortality of approximately $10 \%$, it persists without clinical symptoms for 20 or 30 years. While a proportion of the patients never progress beyond the initial state, a large proportion finally succumbs to the slow but inexorable destruction of the musculature of the heart, the intestines and the esophagus [26]. Finally, Leishmania infections can develop along three different courses:

- A self-limiting, localized cutaneous infection (cutaneous leishmaniasis) that eventually heals and leaves a circular scar, usually on the face. This disease occurs in all of the Middle East, including Afghanistan, as well as Brazil, Colombia, Peru and Bolivia [27];

- Visceral leishmaniasis, which leads to gross hypertrophy of liver and spleen and is mostly fatal. A total of $90 \%$ of visceral leishmaniasis cases occur in India (Bihar), Nepal, Bangladesh, Sudan, Ethiopia and Brazil [28,29];

- The rare mucocutaneous variant in middle and southern America that eventually destroys the mucocutaneous regions of the oral and nasal cavities and leads to death, usually through opportunistic bacterial infections [30].

\section{Chemotherapy}

Chemotherapy of protozoan infections has been grossly neglected by modern pharmacological research. Despite the abundance of protozoan infections and their debilitating consequences, chemotherapy is still mostly based on unsatisfactory and often dated drugs. The situation is particularly unsatisfactory for 'tropical' protozoan infections.

\section{Giardiasis}

For the treatment of uncomplicated giardiasis, numberous compounds are available, including metronidazole (Flagyl $\AA$ ), tinidazol (Fasigyn $®)$ or nitazoxanide (Alinia $®$, Daxon $®$ and Nitax $\left.{ }^{\circledR}\right)$. Despite this relatively comfortable situation, better chemotherapy is still an urgent medical need $[9,31,32]$, particularly because a high recurrence rate after treatment and drug resistance remain major problems $[9,33]$.

\section{Toxoplasmosis}

For toxoplasmosis, most infections occur without overt clinical signs. The infection must be treated in immunocompromised patients. Also, treatment is important if a toxoplasma infection occurs in a naive woman during pregnancy. Here, the infection constitutes a risk for the developing fetus. In addition, the newborn may contract perinatal ocular toxoplasmosis during birth [34]. Early in pregnancy, the macrolide antibiotic spiramycin is often used to minimize the danger of transplacental infection of the fetus. The standard medication later on, as well as for the other types of Toxoplasma infections, is antifolates in combination with folinic acid, although the efficacy of this treatment is still under discussion $[4,35]$. Better and more effective chemotherapy against toxoplasmosis is, therefore, high on the research agenda. 
For malaria, a broad range of compounds from different chemical classes and with different mechanisms of action are available, and research into new drugs and drug targets is ongoing. However, the rapid development of drug resistance by malaria parasites remains a persistent problem [36,37]. The recently reported resistance against the latest antimalarial drug class, the artemisinins, is particularly concerning [38,39]. Artemisinins are considered to interfere with a variety of intracellular targets, and thus development of resistance was considered unlikely. In general, the current situation of drug resistance in malaria is that of an intense competition between the rate of resistance development by the parasite and the efficacy of developing new drugs and treatment concepts. The malaria parasites are genetically haploid during all their stages in the human body. This provides for rapid mutation rates and causes strong selection for resistance whenever they are exposed to drugs at sublethal doses [40]. While this cannot be completely avoided even with the most careful medical practice, the problem is compounded by circumstances that are difficult to control, such as insufficient drug dosing, noncompliance with medication schedules and the abundance of low-quality counterfeit drugs [41].

\section{Chagas disease}

The situation is different, although not more promising, for the diseases caused by kinetoplastid parasites including Chagas disease (T. cruzi), human sleeping sickness (Trypanosoma brucei) and the various leishmaniases (Leishmania spp). These protozoa are diploid and, thus, less prone to acquiring drug resistance. Nevertheless, drug resistance and drug refractoriness are increasing problems in African sleeping sickness and the leishmaniases [42-44]. In addition, and in contrast to the situation for malaria, the armamentarium of available drugs is very limited, and research into developing new compounds has been slow. For Chagas disease, the nitrofurane nifurtimox (Lampit ${ }^{\circledR}$ ) and the nitroimidazole benznidazole (Rochagan ${ }^{\circledR}$ ) are used as standard medication, although their efficacy is suboptimal and they are fraught with serious side effects [45]. The situation is further complicated by the fact that their efficacy against the chronic stage of the infection cannot be evaluated due to the lack of reliable diagnostics of the disease during its latent phase [26].

\section{Sleeping sickness}

For the chemotherapy of human sleeping sickness, there are currently only four or five compounds available. All of them are old (between 20 and 90 years), and all of them are fraught with serious side effects. Among them, suramin (Germanin $®$ ) and pentamidine (Pentacarinat ${ }^{\circledR}$, Pentam ${ }^{\circledR}$ ), are only effective in the early stages of infection, that is, before the parasites have penetrated the CNS. In the late stages of infection, the highly toxic arsenical melarsoprol (Arsobal ${ }^{\circledR}$ ) is used as the standard medication, although the toxicityinduced fatality rate is between 5 and $10 \%$. Resistance to melarsoprol has increased over the last one to two decades [46], which is a major cause for concern. In western Africa, the ornithine decarboxylase inhibitor $\alpha$-difluoro-methyl-ornithine (eflornithine; Ornidyl®) can be used, but its usefulness is seriously restricted because of the complex application (four daily intravenous infusions over $2 \mathrm{~h}$ duration each, for a period of 2 to 3 weeks) and the required logistics. Recently, a nifurtimox-eflornithine combination therapy has been successfully introduced, which may help alleviate these practical problems $[47,48]$. Interesting developments of potential new sleeping sickness medication are still in the experimental stages, including the development of myristoyl-transferase inhibitors [49] or oxaborole 6-carboxamides [50]. Despite a major setback during clinical trials with one lead compound, the development of orally available diamidines still carries great hope and excitement [51,52]. 


\section{Leishmaniases}

For the leishmaniases, pentavalent antimonials have remained the standard medication in recent decades, but drug resistance is becoming an increasing problem in various regions of the world [53]. Although a number of new compounds, such as miltefosine or amphothericin $\mathrm{B}$, have been developed over the last few years [54-56], effective, safe and cost-efficient chemotherapy of the leishmaniases still remains to be developed $[29,43]$.

In summary, the chemotherapy of protozoan infections is still unsatisfactory, despite their enormous influence on human wellbeing and economic productivity in large parts of the world. For some of these diseases, including Chagas disease, human sleeping sickness and the leishmaniases, the development of new and better chemotherapy is an urgent medical need and a challenge for present-day medicinal chemistry.

\section{The 'inverted magic bullet' paradigm}

Traditionally, the development of new antiparasitic drugs has followed one of two paths:

- Chance observations, educated guessing from a medicinal chemistry pointof-view, or repurposing active compounds from other clinical uses. This approach has led to the early identification of trivalent arsenicals as potential anti-trypanosomal drugs, based on initial observations in 1906 by Robert Koch in the field, and followed up by laboratory experimentation by Paul Ehrlich [57,58]. More recently, this general approach to drug discovery was supplemented by numerous in vitro whole-cell screening procedures.

With the onset of the molecular age, this concept was complemented by target-based drug finding strategies. Concerning parasitic protozoa, many of these approaches were implicitly based on Ehrlich's seminal 'magic bullet' concept [58] based on compounds that find their specific (parasite) target without causing harm to the surrounding host tissue.

While this concept has proven spectacularly productive (for example, in the development of antibiotics), it was much less powerful when it came to developing antiprotozoan compounds. The simple reason is that protozoa are genetically and evolutionarily much closer to their human hosts than are the bacteria, which makes avoiding collateral damage to the host by antiparasitic compounds correspondingly more difficult. Nevertheless, numerous highly parasite-specific potential drug targets have been identified in many protozoan proteomes [202] whose practical suitability will now have to be evaluated. Unfortunately, many efforts in developing specific drugs for such targets have been ultimately unsuccessful because they lacked the required financial and technical support. The latter point has proven particularly difficult, since industrial partners were often reluctant to explore 'exotic' parasite-specific drug targets with which they were not acquainted. This reluctance, plus the effort required to develop high-throughput screening (HTS) assays and set up dedicated hitto-lead chemistry programs for discovering inhibitors for poorly investigated enzymes often 'broke the camel's back' and led to the early termination of many such projects. While some of these obstacles are now on the way to being overcome, the translation of basic research on parasite-specific but 'exotic' potential drug targets to industry-scale screening for inhibitors and lead-development programs remains a serious bottleneck.

Hence, the question remains if the high conservation between certain host and parasite enzymes could be turned from an obstacle to a major boon for drug finding. If such a highly conserved human enzyme happens to already be used as a drug target, and both enzyme function and inhibitor development for this enzyme are well explored, and if its parasite homologue is essential for parasite survival, expertise and technology gained with the 
human enzyme could be applied basically as is to developing parasite-selective inhibitors. The parasite-target enzyme could be introduced into already established HTS campaigns without the need to greatly alter standard operating procedures. Once good hits are identified, the medicinal chemistry is already in place to develop hit scaffolds into compounds with the required selectivity to discriminate between the host and the parasite enzymes. This strategy essentially turns the old magic bullet concept upside down: instead of searching for highly parasite-specific, but often biochemically exotic targets, it focuses on enzymes that are highly conserved between humans and the parasites. Of particular interest are human enzymes against which inhibitors are already used as medication for various clinical conditions. The development of inhibitors for the parasite homologues could then build on the vast expertise already available.

\section{Cyclic nucleotide-specific phosphodiesterases: a conserved target for parasite-specific drugs?}

The inverted magic bullet concept has recently been explored in depth using the cyclic nucleotide specific phosphodiesterases (PDEs) as a model class of enzymes. PDEs are highly specific hydrolases that convert the signaling molecules $5^{\prime}, 3^{\prime}$-cyclic adenosine- or guanosine-monophosphate (cAMP or cGMP) into the corresponding $5^{\prime}$-monophosphates (5'AMP and 5'-GMP), and thus are central players in cyclic nucleotide signaling. The human genome codes for 11 PDE families and, due to differential splicing mechanisms, an estimated total of approximately 100 different PDE polypeptides are synthesized and function in different human tissues [59]. Despite this great variety, the catalytic domains of all these PDEs are highly similar, not so much in terms of amino acid sequence (30-40\% amino acid sequence identity) as in terms of their 3D structure (see later discussion). They all belong to the class 1 PDEs that are identifiable by a characteristic signature sequence [60]. In the vertebrates, the class 1 PDEs are the only enzymes capable of efficiently hydrolyzing cyclic nucleotides. In some, but not all unicellular eukaryotes, two further PDEs can exist in addition to the class 1 enzymes, the class 2 PDEs, for example, in the fungi, and class 3 PDEs in Dictyostelium [61].

All human PDEs are current or potential drug targets and are being investigated by numerous research groups based in industry and academia. A number of PDE-inhibitorbased drugs are already on the market for a variety of clinical conditions. These include the PDE3 inhibitor cilostazol (Pletal®; Otsuka Pharma; for intermittent claudication), the PDE4 inhibitor roflumilast (Daxas ${ }^{\circledR}$; Nycomed Pharma; for chronic obstructive pulmonary disease) or the PDE5 inhibitors sildenafil (Viagra®; Pfizer), tadalafil (Cialis®; Lilly ICOS) or vardenafil (Levitra®; Bayer) for erectile dysfunction. Despite the very high structural similarity between the catalytic domains of these and other human PDEs, the clinically used drugs are highly family-selective. This demonstrates that medicinal chemistry has been able to develop inhibitors that are highly selective for the catalytic domains of their respective target PDE, with only minimal effects on the closely related catalytic domains of other PDEs.

\section{The PDEs of T. brucei \& L. major}

Interestingly, the genomes of many protozoan parasites including those of Toxoplasma, Plasmodium or the kinetoplastida T. brucei and L. major contain one or several genes for class 1 PDEs. The genomes of the kinetoplastida code for four different class 1 PDEs and no other PDE classes, similar to the situation in the human genome. All kinetoplastid genomes for which sequence data are currently available contain the same complement of PDE genes [61] (Figure 1), and the respective PDE polypeptides are highly conserved between the species [62]. PDEA codes for a high- $\mathrm{K}_{\mathrm{m}}$, cAMP-specific PDE [63]. PDEB1 and PDEB2 are 
two tandemly arranged genes that code for two highly similar, cAMP-specific PDEs.

Despite their high similarity, their subcellular localization is quite distinct, with TbrPDEB1 localized predominantly in the flagellum, whereas TbrPDEB2 is mainly located within the cell body [64-68]. PDEC codes for a dual-substrate PDE that contains a FYVE domain at its $N$-terminus $[69,70]$. Finally, PDED has been identified only as a DNA sequence.

Comparative sequence analysis of their catalytic domains has demonstrated that they are as closely related to the human PDEs as these are among themselves (Figure 2). For two of these catalytic domains, TbrPDEB1 of $T$. brucei [ [Ke etaL., unpublshed Data] and LmjPDEB1 of $L$. major (see later discussion), $\mathrm{x}$-ray crystallography has recently demonstrated the extensive degree of structural conservation between human and parasite PDEs over large evolutionary distances.

\section{Crystal structures of LmjPDEB1 can serve as templates for inhibitor design Structural architecture}

The catalytic domain of LmjPDEB1 (residues 582-940) showed traceable residues 597-931 in the crystal [71]. The catalytic domain of LmjPDEB1 comprises $16 \alpha$-helices but no $\beta$ strands (Figure 3). Human PDE4 was used as a reference structure since this enzyme is the most thoroughly explored human PDE, and for which the largest number of crystal structures are available. Two divalent metal ions occupy the bottom of the catalytic pocket. Zinc and magnesium were used, without verification, in the structure refinement. The zinc ion coordinates with His-685, His-721, Asp-722, Asp-835 and two bound water molecules. The magnesium ion coordinates with Asp-722 and five water molecules. The octahedral configuration of the two divalent metals in LmjPDEB1 is comparable with those in the human PDEs.

The structure superposition between LmjPDEB1 and human PDEs yielded root-mean-square deviations of 1.3-2.0 $\AA$ for comparable C $\alpha$ atoms of unliganded human PDE1B [72], unliganded human PDE2A [73], human PDE3B liganded with 3-isobutyl-1-methylxanthine (IBMX) [74], PDE4D2-IBMX [75], PDE5A1-IBMX [75], PDE7A1-IBMX [76], PDE9A2IBMX [77] and PDE10A2-cAMP [78]. This comparison indicates a similar topological folding of LmjPDEB1 and the human PDEs.

The superposition revealed that helix H9 of the H-loop (residues 729-754 in LmjPDEB1) and most residues in the M-loop (residues 858-882) of LmjPDEB1 exhibit significant positional shifts of as much as $3 \AA$ for their $\mathrm{C} \alpha$ atoms from those of the human PDE families (Figure 4). This difference is approximately twice the overall root-mean-square deviation between the structures of LmjPDEB1 and human PDEs and is thus statistically significant. Since the H- and M-loops are directly involved in interaction with the inhibitors $[75,79,80]$, their conformational changes appear to have implications on the discovery of parasiteselective inhibitors.

\section{IBMX binding}

3-Isobutyl-1-methylxanthine is a nonselective inhibitor for most human PDE families, but it only weakly inhibits LmjPDEB1 with an $\mathrm{IC}_{50}$ value of $580 \mu \mathrm{M}$. IBMX binds to the active site of LmjPDEB1 with two characteristic interactions that are conserved in most human PDEs [80]: the $\mathrm{O} 6$ atom of the xanthine ring of IBMX forms a hydrogen bond with N\&2 of Gln-887 of LmjPDEB1, and the xanthine ring stacks against Phe-890 of LmjPDEB1 (Figure 4). The binding of IBMX in the LmjPDEB1 structure is similar to that observed in the human PDE-IBMX complexes, although the orientation and position of IBMX show significant differences [78]. These differences may be understandable because the active site pockets of PDEs are much larger than the volume of IBMX. 


\section{A potential parasite pocket for inhibitor binding}

The structural study [71] identified a subpocket in LmjPDEB1, which neighbors the active site of human PDEs and is close to the binding of the cyclopentyl ring of rolipram in human PDE4 (Figure 5). This pocket is made up of residues from the M-loop and helix H14, including Thr-854, Tyr-858, Met-874, Asn-881, Leu-883 and Gly-886 in LmjPDEB1. Two residues, Met-874 and Gly-886 of LmjPDEB1 are located at the entry of the subpocket and appear to serve as gate-keeping residues to isolate the subpocket from the active site. In most human PDE families, the large size of these entry residues isolates the subpocket from the active site [71]. In the LmjPDEB1 structure, a separation of $7.5 \AA$ between the $\mathrm{C} \alpha$ atoms of Met-874 and Gly-886 leaves the subpocket wide open and fully accessible. The corresponding residue Gly-886 is conserved in most other parasite PDEs, except TcrPDEC and TbrPDEC where it is replaced by serine (Figure 5). However, the residues corresponding to Met-874 are glycine in both PDECs, thus still leaving the access to the subpocket open.

Thr- 854 and Tyr-858 of helix H14 and Leu- 883 and Gly-886 of helix H15 form two walls of the pocket, while the fragment around Asn- 881 of the M-loop constitutes the third side of the pocket (Figure 5a). Interestingly, the bottom of the subpocket is open to the molecular surface of LmjPDEB1. Thus, the pocket looks more like an open channel in LmjPDEB1. It has a size capable of accommodating a five-membered ring and shows mixed hydrophilic and hydrophobic characteristics. This subpocket shows variation in size and shape in different parasite PDEs, and we believe that it might be useful for the development of parasite-selective inhibitors.

The experimental demonstration of the extensive structural conservation between the catalytic domains of human PDEs and those of T. brucei and L. major sets the stage for exploiting this extensive similarity by handling parasite PDEs just like yet another human PDE when it comes to setting up HTS and hit-to-lead development programs, without major adjustments to standard procedures required.

\section{TbrPDEB1 \& TbrPDEB2 of $T$. brucei are potential drug targets: genetic validation}

Early observations had indicated that TbrPDEB 1 and TbrPDEB2 might be essential enzymes for bloodstream form T. brucei [68]. This was confirmed by the use of an inducible RNA interference (RNAi) system [65]. A tetracycline-inducible RNAi construct (Figure 6a) directed simultaneously against the TbrPDEB 1 and TbrPDEB 2 was integrated into the genome of bloodstream form $T$. brucei that expressed a bacterial tetracycline repressor. Tetracycline has no effect on wild-type trypanosomes, but it specifically interacts with the bacterial tetracycline repressor expressed by the genetically altered trypanosome strain. In the absence of tetracycline, the tetracycline-repressor binds tightly to its operator DNA, preventing the expression of the double-stranded interfering RNA. The trypanosomes are perfectly viable and cause virulent infections in mice (Figure 6B). However, if the animals receive tetracycline in the drinking water for two days before the infection, or during an ongoing infection, the tetracycline-liganded repressor dissociates from the operator DNA, double-stranded interfering RNA is transcribed, RNAi is induced and the mRNAs of TbrPDEB1 and TbrPDEB2 are rapidly degraded. As a result, trypanosomal PDE activity is strongly diminished, and the intracellular cAMP levels in the trypanosomes rise dramatically, leading to cell lysis. The parasites are rapidly and permanently eliminated from the infected animals (Figure 6B) [65].

\section{Pharmacological validation of parasite PDEs as drug targets}

Trypanosoma cruzi-The T. cruzi genome contains several PDEs (see earlier discussion). From these, only TcrPDEC has been validated as a therapeutic target [76]. The 
enzyme has been described by Kunz et al. as an unusual dual-substrate PDE [64]. It is able to hydrolyze cAMP as well as cGMP, although the biological significance of the latter activity is unclear.

King-Keller et al. synthesized two classes of compounds and screened their inhibitory activity against TcrPDEC and human PDE4D2 [81]. In the catechol diether group, the most interesting inhibitors were found in the series with bulky substituents in the 4-position of the benzene ring; as expected, this chemical class showed decreased activity against HsPDE4. Figure 7 presents two of the most interesting compounds from this study in terms of potency and selectivity.

With both series, a good correlation was found between inhibition of TcrPDEC activity and amastigote growth inhibition in a myoblast assay; inhibiting this enzyme was found to influence the parasite's osmoregulation. Selected compounds were further validated by showing their effect on increasing the intracellular concentration of cAMP in amastigotes.

Leishmania species-A preliminary target validation of the cAMP signaling pathway in L. major was obtained by inhibiting promastigote proliferation and infection of macrophages by high concentrations of a PDE inhibitor (dipyridamole) or by 8-CPT-cAMP [82]. However, because of the high concentrations used and the minimal effects observed, the evidence for this validation is not entirely conclusive.

A series of commercially available PDE inhibitors was tested against the LmjPDEBs [83]. Only dipyridamole, trequinsin and etazolate caused some inhibition with $\mathrm{IC}_{50}$ values in the range of 10 to $100 \mu \mathrm{M}$. The three inhibitors also inhibited the proliferation of $L$. major promastigotes and Leishmania infantum amastigotes in culture, all with $\mathrm{IC}_{50}$ values in the $30-100 \mu \mathrm{M}$ range. Despite the high inhibitor concentrations required in these experiments, the results clearly suggest the validity of the Leishmania PDEBs as therapeutic targets, as was found for T. brucei ([65]; see earlier discussion).

For $\mathrm{LmjPDEB} 1$, an $\mathrm{IC}_{50}$ value of $580 \mu \mathrm{M}$ for the nonselective PDE inhibitor IBMX was reported ([71]; see earlier discussion). A number of other compounds inhibited the enzyme $>50 \%$ at $20 \mu \mathrm{M}$, including NVP (a specific inhibitor of human PDE4), dipyridamole and the flavonoids luteolin and quercetin (Figure $8 \&$ table 1 [T. brucei]).

Plasmodium species-P. falciparum is the only protozoan pathogen apart from $T$. brucei and $L$. major for which the PDEs are at least marginally explored. Its genome encodes four class 1 PDEs $[61,84,85]$. Yuasa et al. [86] and Wentzinger et al. [84] described PfPDE $\alpha$ as a cGMP-selective PDE. While the deletion of the gene for PfPDE $\alpha$ has no observable effect on the proliferation of $P$. falciparum in culture [84], deletion of PfPDE $\beta$ disrupts gametocytogenesis [87], and deletion of the PfPDE $\delta$ homologue in the mouse malaria agent $P$. berghei interferes with gliding motility, ookinete morphology and parasite transmission [88]. These observations indicate that, in analogy to the situation seen with $T$. brucei, inhibition of plasmodial PDEs might well kill the parasite and, through the disruption of normal gametocytogenesis via PfPDE $\beta$ inhibition, also interrupt the transmission cycle.

Several known PDE inhibitors were tested against PfPDE $\alpha$, and zaprinast, a known selective inhibitor of human PDE5 turned out to be the most potent, with an $\mathrm{IC}_{50}$ value of $3.8 \mu \mathrm{M}$. In contrast, the much more potent (against human PDE5) inhibitor sildenafil showed much lower activity against PfPDE $\alpha\left(\mathrm{IC}_{50}=56 \mu \mathrm{M}\right)$. 
This difference between sildenafil and zaprinast already shows the considerable differences in the structure-activity relationship between PfPDE $\alpha$ and HsPDE5. This observation seems to indicate that selective optimization of the PfPDE $\alpha$ inhibiting activity should be possible in this class of compounds. Target validation of PfPDE $\alpha$ is further supported by the observation that zaprinast $\left(\mathrm{EC}_{50}=35 \mu \mathrm{M}\right.$; [86]), 8-Br-cGMP $\left(\mathrm{EC}_{50}=6.25-12.5 \mu \mathrm{M}\right)$ and 8$\mathrm{Br}-\mathrm{cAMP}\left(\mathrm{EC}_{50}=3.13-6.25 \mu \mathrm{M}\right.$ [101] $)$ inhibit the growth of a plasmodium strain $(P$. falciparum Honduras-1) in vitro.

In a patent application from the Institute Pasteur and the University of Lille (France) [102], a series of $\beta$-carbolines were described with potent activity against different $P$. falciparum strains in an in vitro proliferation assay. This class of compounds comprises analogues of the potent and selective PDE5 inhibitor, tadalafil (Cialis $\left.{ }^{\circledR}\right)$. However tempting it may be to ascribe the effect of these compounds to PDE inhibition, no proof for this mechanism has been given, such as a correlation between the effect on Plasmodium proliferation and changes in intracellular cAMP or cGMP. Compound 70c in this study is an example that showed approximate equipotency against three different strains of $P$. falciparum in vitro ( $\mathrm{IC}_{50}$ values: $\mathrm{GHA}=0.2 \mu \mathrm{g} / \mathrm{ml} ; 3 \mathrm{D} 7=0.13 \mu \mathrm{g} / \mathrm{ml} ; \mathrm{K} 1=0.15 \mu \mathrm{g} / \mathrm{ml}$ ). Interestingly, some of the compounds from this patent application were also active in vitro against T.b.

rhodesiense, with compound 28 being the most active with an $\mathrm{IC}_{50}$ value of $0.1 \mu \mathrm{g} / \mathrm{ml}$.

Trypanosoma brucei-Besides the genetic validation of TbrPDEB1 and TbrPDEB2 as drug targets in $T$. brucei (see earlier discussion), some preliminary pharmacological validation has been performed. Several known PDE inhibitors have been shown to inhibit both cell proliferation and the TbrPDEB enzymes (IBMX, sildenafil, dipyridamole, etazolate, ethaverine and trequinsin), although with low potency $[68,89]$. These results, as well as the availability of $\mathrm{x}$-ray crystallographic structures for at least two catalytic domains of kineto-plastid PDEs has sparked activities towards exploring PDE inhibitors as potential new trypanocidal drugs.

X-ray crystallography data (see earlier discussion) have demonstrated the high degree of structural conservation between the catalytic domains of human and parasite PDEs. On the other hand, they have shown the presence of a pocket that is connected to the substratebinding pocket, but can only be accessed from there in the parasite PDEs, but not in the human PDEs. The structural comparison between LmjPDEB1 and human PDE4D suggested that the scaffold of human PDE inhibitors might be useful for the development of parasiteselective inhibitors. A model docking shows that the scaffold of the catechol part of rolipram is seemingly a good starting model (Figure 9a). In an attempt to improve parasite-versushost specificity, a long and flexible arm was designed to extend the catechol of the inhibitor towards the P-pocket. This strategy provided encouraging results. The compound synthesized in Ke's laboratory, University of North Carolina at Chapel Hill (USA), showed an $\mathrm{IC}_{50}$ value of $3.1 \mu \mathrm{M}$ for the catalytic domain of LmjPDEB1 and 4.6 $\mu \mathrm{M}$ for TbrPDEB1. Further optimization is in progress. The available crystallographic data will now form the groundwork for further medicinal chemistry.

The biological validation of $T$. brucei PDEs as drug targets has been followed up by HTS of Nycomed Pharma's proprietary library of 400,000 compounds. Hit-to-lead development of scaffolds recovered in the screen, as well as of other leads, is now in progress. In addition, new exploration of chemical space by fragment-based screening is also under way within the framework of the TI Pharma T4-302 consortium [203]. 


\section{Conclusion}

The inverted silver bullet concept postulates that suitable parasite-specific drug targets need not necessarily be highly parasite-specific enzymes or other components of parasite-specific metabolic pathways. Quite on the contrary, many occasions it might be preferable to target a parasite enzyme with highly conserved homologues in the human host. If this conserved human target has already been extensively explored and exploited as a drug target for other clinical conditions, and specific inhibitors for the human target have been developed, then the established technology can be applied without major modifications to developing compounds that inhibit the closely related parasite homologue. Issues of host-versus-parasite specificity can be confidently taken care of by medicinal chemistry. This approach will allow mustering the vast expertise and technology of the pharmaceutical industry to address parasitic diseases, without the need to retool, rethink or reinvent the entire drug screening and development process.

The PDEs of $T$. brucei and L. major are suitable targets to explore this concept. They are as conserved with respect to their human homologues as these eleven human PDE families are among themselves. Nevertheless, in human pharmacology, highly selective PDE inhibitors have been developed that potently inhibit one PDE family with minimal crosstalk to PDEs of others. Given the extensive similarity between human and trypanosomal PDEs, a similar degree of specificity should also be obtainable for inhibitors of the parasite PDEs.

X-ray crystallography of the catalytic domains of LmjPDEB1 has shown that there are sufficient differences in the detailed structures of human and Leishmania PDEs to leave room for chemical improvement of potency and specificity of lead compounds. The availability of structural data will now considerably speed up this chemical development.

For T. brucei, experimental data have demonstrated that TbrPDEB1 and TbrPDEB2 are essential enzymes, and that their inactivation by either pharmacological means or by RNAi leads to rapid cell death in vitro, and to a complete halt of ongoing infections in a mouse model. Similarly, experimental evidence from the human malaria parasite $P$. falciparum and the mouse parasite Plasmodium berghei strongly indicate that interfering with these parasites' PDEs may also interrupt the infection. Similar evidence for other protozoan parasites is still lacking, but the experimental tools are available to follow suit.

The remaining problems with developing PDE inhibitors into antiparasite drugs should not be underestimated. Besides establishing that PDEs are, in fact, valid drug targets, the potential interaction of antiparasite PDE inhibitors with human PDEs has to be worked out for every single case, and the side effects of such inhibitions due to inhibitor crosstalk to human PDEs remains to be assessed. Nevertheless, the history of developing inhibitors against individual human PDEs has amply demonstrated that exquisite specificity for a given human PDE can be obtained, with a negligible effect of an inhibitor of one PDE to any of the other ten human isoenzymes. However, tailoring an inhibitor for optimal potency and specificity inevitably makes it more prone for inducing drug resistance by spontaneous, single-point mutations of the PDE. This is not a problem in all current applications of PDE inhibitors, such as chronic obstructive pulmonary disease (PDE4 inhibitors) or erectile dysfunction (PDE 5 inhibitors). In contrast, drug resistance certainly will occur once PDE inhibitors are used for treating protozoal infections. However, this problem is not specific for PDE inhibitors, as the long and frustrating experience with drug-resistant parasites amply documents. Drug resistance will have to be approached by means other than medicinal chemistry, including the exclusive use of combination drugs, strict enforcement of drug quality standards also in retail outlets, and a judicious use of the available drugs. 
The generic approach outlined in this study targets parasite enzymes that are highly conserved homologues of human enzymes that are already extensively studied as drug targets for a variety of clinical conditions. This strategy offers the unique advantage that well-established developmental pipelines and standard procedures can also be applied to develop new anti-parasitic drugs. This repurposing will considerably lower the required financial and intellectual investments that any company will have to risk when considering the development of antiparasitic drugs. While this alone might already make such an undertaking considerably more attractive, an increasing number of public-private and product-development partnerships [90,201,203-206] serve to facilitate all aspects of drug development and the cooperation and communication between industry and academia. In addition, a number of creative financial and regulatory schemes have been developed over the years to facilitate and accelerate (and ultimately reward) the development of urgently required drugs against neglected diseases [91,92].

Despite the inevitable remaining obstacles, the case of the PDEs in trypanosomes and Leishmania has demonstrated the viability of the inverted silver bullet concept. In view of the fact that the genomes of humans and their protozoan parasites contain a fair number of highly conserved enzymes, which are well studied drug targets in the human, this approach might hasten the development of urgently needed new antiparasitic drugs. Hopefully, this eventually might lead to what Paul Ehrlich envisioned in his 1908 Nobel lecture, namely that "...It is really possible in every animal species and with every kind of trypanosome infection to achieve a complete cure with one injection, a result which corresponds to what I call therapia sterilisans magna" [207].

\section{Future perspective}

Over the next decade or so, chemotherapy of protozoal diseases will gain importance for several reasons:

- The global exchange of people and disease vectors, in combination with the projected global warming trends will establish new habitats for protozoal parasites in areas where they are currently absent or insignificant;

- For some protozoa, including malaria, the projections indicate an increase in prevalence, while for others, such as Trichomonas, their significance as a public health problem is only becoming visible now;

- The long-term economic development of many major countries will enable them to allocate more funds to public health problems, including the treatment and prevention of parasitic diseases;

- The increasing scientific competence and political influence of many endemic countries will gradually shift the global research agenda towards addressing problems that are of major concern to them. These will prominently include the control, prevention and chemotherapy of infectious diseases.

On the other hand, due to the genetic flexibility of protozoal parasites, the development of antiprotozoal drug will never be over. Rather, we are locked in an endless race between the competence and creativity of drug developers and the genetic flexibility and evolutionary pressure of protozoal parasites. Thus, the quest for new drug targets and new drug scaffolds will remain a continuous effort.

The field might strongly benefit from newer technical developments, such as the current deep-sequencing revolution. Eventually, such techniques might permit a much more precise definition of the genotype of a parasite strain in a given locality or even a given patient, 
hopefully allowing a much more effective selection and dosing of drugs. This would also go a long way towards reducing the development of drug resistance.

Hopefully, in the long term we might finally learn and, importantly, also apply the lessons learned of how to prevent parasite infections or reduce vector levels in the first place. A salient example are the intestinal parasites that could, at least in theory, be completely eliminated as human-disease agents simply by providing clean drinking water, with no need of expensive medication or vaccination. Here, the lesson is clear for all to see, and the great William Trager of Rockefeller University (USA) formulated it so well in 1986 by stating that "good plumbing has done more for good health than has good medicine". This is a timely reminder that we should not too narrowly focus our efforts on pharmacological solutions, but always remain open to the fact that combating protozoal pathogens is a multidimensional task where single or simple solutions are bound to fail.

\section{Acknowledgments}

Financial \& competing interests disclosure

This work was supported by NIH grant GM59791 (to HK), by grant Nr 3100A-109245 of the Swiss National Science Foundation (to TS) and by the TI Pharma consortium T4-304. The authors have no other relevant affiliations or financial involvement with any organization or entity with a financial interest in or financial conflict with the subject matter or materials discussed in the manuscript apart from those disclosed. No writing assistance was utilized in the production of this manuscript.

\section{Bibliography}

Papers of special note have been highlighted as:

- of interest

m of considerable interest

1. Barratt JL, Harkness J, Marriott D, Ellis JT, Stark D. Importance of nonenteric protozoan infections in immunocompromised people. Clin. Microbiol. Rev. 2010; 23(4):795-836. [PubMed: 20930074]

2. Wiser, MF. Garland Science. Taylor and Francis; London, UK: 2010. Protozoa and Human Disease.

3. Cook, GC.; Shetty, NP.; Shetty, PS., et al. Manson's Tropical Diseases. 22nd Edition. Cook, GC.; Zumla, A., editors. Academic Press; NY, USA: 2009.

4. Montoya JG, Remington JS. Management of Toxoplasma gondii infection during pregnancy. Clin. Infect. Dis. 2008; 47(4):554-566. [PubMed: 18624630]

5. Commodaro AG, Belfort RN, Rizzo LV, et al. Ocular toxoplasmosis: an update and review of the literature. Mem. Inst. Oswaldo Cruz. 2009; 104(2):345-350. [PubMed: 19430662]

6. Van Der Pol B, Kwok C, Pierre-Louis B, et al. Trichomonas vaginalis infection and human immunodeficiency virus acquisition in African women. J. Infect. Dis. 2007; 197(4):548-554. [PubMed: 18275275]

7. Plutzer J, Ongerth J, Karanis P. Giardia taxonomy, phylogeny and epidemiology: facts and open questions. Int. J. Hyg. Environ. Health. 2010; 213(5):321-333. [PubMed: 20619729]

8. Goodgame RW. Understanding intestinal spore-forming protozoa: cryptosporidia, microsporidia, isospora, and cyclospora. Ann. Intern. Med. 1996; 124(4):429-441. [PubMed: 8554253]

9. Lalle M. Giardiasis in the post genomic era: treatment, drug resistance and novel therapeutic perspectives. Infect. Disord. Drug Targets. 2010; 10(4):283-294. [PubMed: 20429863]

10. Petry F, Jakobi V, Tessema TS. Host immune response to Cryptosporidium parvum infection. Exp. Parasitol. 2010; 126(3):304-309. [PubMed: 20685209]

11. Vijayan VK, Kilani T. Emerging and established parasitic lung infestations. Infect. Dis. Clin. North Am. 2010; 24(3):579-602. [PubMed: 20674793] 
12. Stark D, Barratt JL, van Hal S, Marriott D, Harkness J, Ellis JT. Clinical significance of enteric protozoa in the immunosuppressed human population. Clin. Microbiol. Rev. 2009; 22(4):634-650. [PubMed: 19822892]

13. Sabbatani S, Fiorino S, Manfredi R. The emerging of the fifth malaria parasite (Plasmodium knowlesi): a public health concern? Braz. J. Infect. Dis. 2010; 14(3):299-309. [PubMed: 20835518]

14. Cox-Singh J, Davis TM, Lee KS, et al. Plasmodium knowlesi malaria in humans is widely distributed and potentially life threatening. Clin. Infect. Dis. 2008; 46(2):165-171. [PubMed: 18171245] — The serendipitous discovery of an unexpected, new human pathogen.

15. White NJ. Plasmodium knowlesi: the fifth human malaria parasite. Clin. Infect. Dis. 2008; 46:172173. Editorial Commentary. [PubMed: 18171246]

16. Feachem RG, Phillips AA, Hwang J, et al. Shrinking the malaria map: progress and prospects. Lancet. 2010; 376(9752):1566-1578. [PubMed: 21035842]

17. Sachs J, Malaney P. The economic and social burden of malaria. Nature. 2002; 415(6872):680685. [PubMed: 11832956] @ Provides the 'greater picture' of the effects of malaria from economic, political and developmental points of view.

18. Stanley SL Jr. Amoebiasis. Lancet. 2003; 361(9362):1025-1034. [PubMed: 12660071]

19. Ximenez C, Moran P, Rojas L, Valadez A, Gomez A. Reassessment of the epidemiology of amebiasis: state of the art. Infect. Genet. Evol. 2010; 9(6):1023-1032. [PubMed: 19540361]

20. Marin C, Dollet M, Pagès M, Bastien P. Large differences in the genome organization of different plant Trypanosomatid parasites (Phytomonas ssp.) reveal wide evolutionary divergences between taxa. Infect. Genet. Evol. 2009; 9(2):235-240. [PubMed: 19111630]

21. Liao D, Shen J. Studies of quinapyramine-resistance of Trypanosoma brucei evansi in China. Acta Trop. 2010; 116(3):173-177. [PubMed: 20813092]

22. Gutierrez C, Corbera JA, Morales M, Büscher P. Trypanosomosis in goats: current status. Ann. NY Acad. Sci. 2006; 1081:300-310. [PubMed: 17135529]

23. Tibary A, Fite C, Anouassi A, Sghiri A. Infectious causes of reproductive loss in camelids. Theriogenology. 2006; 66(3):633-647. [PubMed: 16697037]

24. Boelaert M, Meheus F, Robays J, Lutumba P. Socio-economic aspects of neglected diseases: sleeping sickness and visceral leishmaniasis. Ann. Trop. Med. Parasitol. 2010; 104(7):535-542. [PubMed: 21092391]

25. Brun R, Blum J, Chappuis F, Burri C. Human African trypanosomiasis. Lancet. 2010; 375(9709): 148-159. [PubMed: 19833383] — Up-to-date overview of African trypanosomiasis.

26. Lescure FX, Le Loup G, Freilij H, et al. Chagas disease: changes in knowledge and management. Lancet Infect. Dis. 2010; 10(8):556-570. [PubMed: 20670903]

27. Ameen M. Cutaneous leishmaniasis: advances in disease pathogenesis, diagnostics and therapeutics. Clin. Exp. Dermatol. 2010:699-705. [PubMed: 20831602]

28. Blackwell JM, Fakiola M, Ibrahim ME, et al. Genetics and visceral leishmaniasis: of mice and man. Parasite Immunol. 2009; 31(5):254-266. [PubMed: 19388946]

29. Chappuis F, Sundar S, Hailu A, et al. Visceral leishmaniasis: what are the needs for diagnosis, treatment and control? Nature Rev. Microbiol. 2007; 5(11):873-882. [PubMed: 17938629]

30. David CV, Craft N. Cutaneous and mucocutaneous leishmaniasis. Dermatol. Ther. 2009; 22(6): 491-502. [PubMed: 19889134]

31. Tian HF, Chen B, Wen JF. Giardiasis, drug resistance, and new target discovery. Infect. Disord. Drug Targets. 2010; 10(4):295-302. [PubMed: 20429862]

32. Zaat JO, Mank TH, Assendelft WJ. Drugs for treating giardiasis. Withdrawn. Cochrane Database Syst. Rev. 2007; 18(2):CD000217. [PubMed: 17636622]

33. Wright JM, Dunn LA, Upcroft P, Upcroft JA. Efficacy of antigiardial drugs. Expert Opin. Drug. Saf. 2003; 2(6):529-541. [PubMed: 14585063]

34. Talabani H, Mergey T, Yera H, et al. Factors of occurrence of ocular toxoplasmosis. Parasite. 2010; 17(3):177-182. A review. [PubMed: 21073138]

35. Stanford MR, Gilbert RE. Treating ocular toxoplasmosis: current evidence. Mem. Inst. Oswaldo Cruz. 2009; 104(2):312-315. [PubMed: 19430659] 
36. Dondorp AM, Yeung S, White L, et al. Artemisinin resistance: current status and scenarios for containment. Nat. Rev. Microbiol. 2010; 8(4):272-280. [PubMed: 20208550]

37. Naidoo I, Roper C. Following the path of most resistance: dhps K540E dispersal in African Plasmodium falciparum. Trends Parasitol. 2010; 26(9):447-456. [PubMed: 20728060]

38. Jambou R, Legrand E, Niang M, et al. Resistance of Plasmodium falciparum field isolates to invitro artemether and point mutations of the SERCA-type PfATPase6. Lancet. 2005; 366(9501): 1960-1963. [PubMed: 16325698]

39. Noedl H, Se Y, Schaecher K, et al. Evidence of artemisinin-resistant malaria in western Cambodia. N. Engl. J. Med. 2008; 359(24):2619-2620. [PubMed: 19064625]

40. Mackinnon MJ, Marsh K. The selection landscape of malaria parasites. Science. 2010; 328(5980): 866-871. [PubMed: 20466925]

41. Newton PN, Green MD, Fernández FM, Day NP, White NJ. Counterfeit anti-infective drugs. Lancet Infect. Dis. 2006; 6(9):602-613. [PubMed: 16931411]

42. Gehrig S, Efferth T. Development of drug resistance in Trypanosoma brucei rhodesiense and Trypanosoma brucei gambiense. Treatment of human African trypanosomiasis with natural products (review). Int. J. Mol. Med. 2008; 22(4):411-419. [PubMed: 18813846]

43. Santos DO, Coutinho CE, Madeira MF, et al. Leishmaniasis treatment - a challenge that remains: a review. Parasitol. Res. 2008; 103(1):1-10. [PubMed: 18389282]

44. Matovu E, Seebeck T, Enyaru JC, Kaminsky R. Drug resistance in Trypanosoma brucei spp., the causative agents of sleeping sickness in man and nagana in cattle. Microbes Infect. 2001; 3(9): 763-770. [PubMed: 11489425]

45. Apt W. Current and developing therapeutic agents in the treatment of Chagas disease. Drug Des. Devel. Ther. 2010; 4:243-253.

46. De Koning HP. Ever-increasing complexities of diamidine and arsenical crossresistance in African trypanosomes. Trends Parasitol. 2008; 24(8):345-349. [PubMed: 18599351]

47. Yun O, Priotto G, Tong J, Flevaud L, Chappuis F. NECT is next: implementing the new drug combination therapy for Trypanosoma brucei gambiense sleeping sickness. PLoS Negl. Trop. Dis. 2010; 4(5):e720. [PubMed: 20520803]

48. Jacobs RT, Nare B, Phillips MA. State of the art in African trypanosome drug discovery. Curr. Top. Med. Chem. 2011; 11(10):1255-1274. [PubMed: 21401507] @ Up-to-date review of the current state of chemotherapy and drug development.

49. Frearson JA, Brand S, McElroy SP, et al. $N$-myristoyltransferase inhibitors as new leads to treat sleeping sickness. Nature. 2010; 646(7289):728-732. [PubMed: 20360736]

50. Nare B, Wring S, Bacchi C, et al. Discovery of novel orally bioavailable oxaborole 6carboxamides that demonstrate cure in a murine model of late-stage central nervous system african trypanosomiasis. Antimicrob. Agents Chemother. 2010; 54(10):4379-4388. [PubMed: 20660666] m Example of a straightforward drug-development program based on a new scaffold.

51. Soeiro MN, de Castro SL, de Souza EM, Batista DG, Silva CF, Boykin DW. Diamidine activity against trypanosomes: the state of the art. Curr. Mol. Pharmacol. 2008; 1(2):151-161. [PubMed: 20021429]

52. Wenzler T, Boykin DW, Ismail MA, Hall JE, Tidwell RR, Brun R. New treatment option for second-stage African sleeping sickness: in vitro and in vivo efficacy of aza analogs of DB289. Antimicrob. Agents Chemother. 2009; 53(10):4185-4192. [PubMed: 19620327]

53. Croft SL, Sundar S, Fairlamb AH. Drug resistance in leishmaniasis. Clin. Microbiol. Rev. 2006; 19(1):111-126. [PubMed: 16418526]

54. Goto H, Lindoso JA. Current diagnosis and treatment of cutaneous and mucocutaneous leishmaniasis. Expert Rev. Anti Infect. Ther. 2010; 8(4):419-433. [PubMed: 20377337]

55. Richard JV, Werbovetz KA. New antileishmanial candidates and lead compounds. Curr. Opin. Chem. Biol. 2010; 14(4):447-455. [PubMed: 20400358]

56. Croft SL. Kinetoplastida: new therapeutic strategies. Parasite. 2008; 15(3):522-527. [PubMed: 18814734]

57. Schwartz RS. Paul Ehrlich's magic bullets. N. Engl. J. Med. 2004; 350(11):1079-1080. [PubMed: 15014180] 
58. Winau F, Westphal O, Winau R. Paul Ehrlich - in search of the magic bullet. Microbes Infect. 2004; 6:786-789. [PubMed: 15207826]

59. Aandahl, EM.; Abi-Gerges, A.; Beavo, JA., et al. Cyclic Nucleotide Phosphodiesterases. In: Beavo, JA.; Francis, S.; Houslay, M., editors. Health and Disease. CRC Press; Boca Raton, FL, USA: 2007.

60. Beavo, JA.; Houslay, MD.; Francis, SH. Cyclic nucleotide phosphodiesterase superfamily. In: Beavo, JA.; Francis, S.; Houslay, M., editors. Cyclic Nucleotide Phosphodiesterases in Health and Disease. CRC Press; Boca Raton, FL, USA: 2007. Chapter 1

61. Wentzinger, L.; Seebeck, T. Protozoal phosphodiesterases. In: Beavo, JA.; Francis, S.; Houslay, M., editors. Cyclic Nucleotide Phosphodiesterases in Health and Disease. CRC Press; Boca Raton, FL, USA: 2007. Chapter 14

62. Kunz S, Beavo JA, D’Angelo MA, et al. Cyclic nucleotide specific phosphodiesterases of the kinetoplastida: a unified nomenclature. Mol. Biochem. Parasitol. 2006; 145(1):133-135. [PubMed: 16280178]

63. Kunz S, Kloeckner T, Essen LO, Seebeck T, Boshart M. TbPDE1, a novel class 1 phosphodiesterase of Trypanosoma brucei. Eur. J. Biochem. 2004; 271:637-647. [PubMed: 14728691]

64. Luginbuehl E, Ryter D, Schranz-Zumkehr J, Oberholzer M, Kunz S, Seebeck T. The N-terminus of phosphodiesterase TbrPDEB1 of Trypanosoma brucei contains the signal for integration into the flagellar skeleton. Eukaryotic Cell. 2010; 9:1466-1475. [PubMed: 20693305]

65. Oberholzer M, Marti G, Baresic M, Kunz S, Hemphill A, Seebeck T. The Trypanosoma brucei cAMP phosphodiesterasesTbrPDEB1 and TbrPDEB2: flagellar enzymes that are essential for parasite virulence. FASEB J. 2007; 21:720-731. [PubMed: 17167070] @ In vivo demonstration that phosphodiesterases (PDEs) are essential for trypanosome infectivity and survival in mouse.

66. D’Angelo MA, Sanguineti S, Reece JM, Birnbaumer L, Torres HN, Flawiá MM. Identification, characterization and subcellular localization of TcPDE1, a novel cAMP-specific phosphodiesterase from Trypanosoma cruzi. Biochem. J. 2004; 378(1):63-72. [PubMed: 14556647]

67. Rascón A, Soderling SH, Schaefer JB, Beavo JA. Cloning and characterization of a cAMP-specific phosphodiesterase (TbPDE2B) from Trypanosoma brucei. Proc. Natl Acad. Sci. USA. 2002; 99(7):4714-4719. [PubMed: 11930017]

68. Zoraghi R, Seebeck T. The cAMP-specific phosphodiesterase TbPDE2C is an essential enzyme in bloodstream form Trypanosoma brucei. Proc. Natl Acad. Sci. USA. 2002; 99:4343-4348. [PubMed: 11930001]

69. Kunz S, Oberholzer M, Seebeck T. A FYVE-containing unusual cyclic nucleotide phosphodiesterase from Trypanosoma brucei. FEBS J. 2005; 272:6412-6422. [PubMed: 16336277]

70. Schoijet AC, Miranda K, Medeiros LC, et al. Defining the role of a FYVE domain in the localization and activity of a cAMP phosphodiesterase implicated in osmoregulation in Trypanosoma cruzi. Mol. Microbiol. 2010; 79(1):50-62. [PubMed: 21166893]

71. Wang H, Yan Z, Geng J, Kunz S, Seebeck T, Ke H. Crystal structure of the Leishmania majorphosphodiesterase LmjPDEB1 and insight into the design of the parasite-selective inhibitors. Mol. Microbiol. 2007; 66(4):1029-1038. [PubMed: 17944832] — First structure of a parasite PDE. Demonstrates strong overall similarity with human PDE4, and identifies a parasite-specific pocket.

72. Zhang KY, Card GL, Suzuki Y, et al. A glutamine switch mechanism for nucleotide selectivity by phosphodiesterases. Mol. Cell. 2004; 15(2):279-286. [PubMed: 15260978]

73. Iffland A, Kohls D, Low S, et al. Structural determinants for inhibitor specificity and selectivity in PDE2A using the wheat germ in vitro translation system. Biochemistry. 2005; 44(23):8312-8325. [PubMed: 15938621]

74. Scapin G, Patel SB, Chung C, et al. Crystal structure of human phosphodiesterase 3B: atomic basis for substrate and inhibitor specificity. Biochemistry. 2004; 43(20):6091-6100. [PubMed: 15147193] 
75. Huai Q, Liu Y, Francis SH, Corbin JD, Ke H. Crystal structures of phosphodiesterases 4 and 5 in complex with inhibitor IBMX suggest a conformation determinant of inhibitor selectivity. J. Biol. Chem. 2004; 279(13):13095-13101. [PubMed: 14668322]

76. Wang H, Liu Y, Chen Y, Robinson H, Ke H. Multiple elements jointly determine inhibitor selectivity of cyclic nucleotide phosphodiesterases 4 and 7. J. Biol. Chem. 2005; 280(35):3094930955. [PubMed: 15994308]

77. Huai Q, Wang H, Zhang W, Colman RW, Robinson H, Ke H. Crystal structure of phosphodiesterase 9 in complex with inhibitor IBMX. Proc. Natl Acad. Sci. USA. 2004; 101(26): 9624-9629. [PubMed: 15210993]

78. Wang H, Liu Y, Hou J, Zheng M, Robinson H, Ke H. Structural insight into substrate specificity of phosphodiesterase 10. Proc. Natl Acad. Sci. USA. 2007; 104(14):5782-5787. [PubMed: 17389385]

79. Wang H, Liu Y, Huai Q, et al. Multiple conformations of phosphodiesterase-5: implications for enzyme function and drug development. J. Biol. Chem. 2006; 281(30):21469-21479. [PubMed: 16735511]

80. Ke H, Wang H. Crystal structures of phosphodiesterases and implications on substrate specificity and inhibitor selectivity. Curr. Top. Med. Chem. 2007; 7(4):391-403. [PubMed: 17305581] Overview of the structures of PDE catalytic domains and their substrate-inhibitor interactions.

81. King-Keller S, Li M, Smith A, et al. Chemical validation of phosphodiesterase C as a chemotherapeutic target in Trypanosoma cruzi, the etiological agent of Chagas' disease. Antimicrob. Agents Chemother. 2010; 54(9):3738-3745. [PubMed: 20625148]

82. Malki-Feldman L, Jaffe CL. Leishmania major: effect of protein kinase A and phosphodiesterase activity on infectivity and proliferation of promastigotes. Exp. Parasitol. 2009; 123(1):39-44. [PubMed: 19463817]

83. Johner A, Kunz A, Linder M, Shakur Y, Seebeck T. Cyclic nucleotide specific phosphodiesterases of Leishmania major. BMC Microbiol. 2006; 6:25. [PubMed: 16522215]

84. Wentzinger L, Bopp S, Tenor H, et al. Cyclic nucleotide-specific phosphodiesterases of Plasmodium falciparum: PfPDE $\alpha$, a non-essential cGMP-specific PDE that is an integral membrane protein. Int. J. Parasitol. 2008; 38:1625-1637. [PubMed: 18590734]

85. Young JA, Fivelman QL, Blair PL, et al. The Plasmodium falciparum sexual development transcriptome: a microarray analysis using ontology-based pattern identification. Mol. Biochem. Parasitol. 2005; 143(1):67-79. [PubMed: 16005087]

86. Yuasa K, Mi-Ichi F, Kobayashi T, et al. PfPDE1, a novel cGMP-specific phosphodiesterase from the human malaria parasite Plasmodium falciparum. Biochem. J. 2005; 392(1):221-229. [PubMed: 16038615]

87. Taylor CJ, McRobert L, Baker DA. Disruption of a Plasmodium falciparum cyclic nucleotide phosphodiesterase gene causes aberrant gametogenesis. Mol. Microbiol. 2008; 69(1):110-118. [PubMed: 18452584]

88. Moon RW, Taylor CJ, Bex C, et al. A cyclic GMP signalling module that regulates gliding motility in a malaria parasite. PLoS Pathog. 2009; 5(9):e1000599. [PubMed: 19779564]

89. Zoraghi R, Kunz S, Gong KW, Seebeck T. Characterization of TbPDE2A, a novel cyclic nucleotide specific phosphodiesterase from the protozoan parasite Trypanosoma brucei. J. Biol. Chem. 2001; 276(15):11559-11566. [PubMed: 11134002]

90. Butler D. Neglected diseases fund touted. Nature. 2010; 465(7296):277. [PubMed: 20485404]

91. Cole P. Accelerating drug development and approval. Drug News Perspect. 2010; 23(1):37-47. [PubMed: 20155218]

92. Waltz E. FDA launches priority vouchers for neglected-disease drugs. Nat Biotechnol. 2008; 26(12):1315-1316. [PubMed: 19060849]

93. Carson M. Ribbons. Methods Enzymol. 1997; 277:493-505. [PubMed: 18488321]

94. Motyka SA, Englund PT. RNA interference for analysis of gene function in trypanosomatids. Curr. Opin. Microbiol. 2004; 7(4):362-368. [PubMed: 15288622] 


\section{$\square$ Patents}

101. Tanabe Seiyaku Co., Ltd. WO044192. 2004.

102. Universite de Lille. WO044144. 2008.

\section{$\square$ Websites}

201. Roll Back Malaria Partnership. Document number RBM/SEC/2009/ISS.2. www.rollbackmalaria.org

202. The TDR Targets Database. www.tdrtargets.org

203. TI Pharma. www.tipharma.com

204. TriTryp Database. www.tritrypdb.org

205. Drugs for Neglected Diseases Initiative. www.dndi.org

206. Bill and Melinda Gates Foundation. www.gatesfoundation.org/Pages/home.aspx

207. The Nobel Prize in Physiology and Medicine.

http://nobelprize.org/nobel_prizes/medicine/laureates/1908/ehrlich-lecture.html

208. OneWorld Health. www.oneworldhealth.org 
Executive summary

- Protozoan parasites remain a huge, unsolved problem in large parts of our world. Much effort is currently devoted to controlling transmission (e.g., through reduction of the relevant insect vectors or improvement of drinking water quality) or to the development of vaccines. Nevertheless, chemotherapy remains an important pillar of disease control.

- Despite the significance of protozoan infections, chemotherapy of these diseases has not kept pace with modern medicinal chemistry. For most of these diseases, only unsatisfactory drugs are available.

- The development of such parasite-specific drugs has been hampered by the lack of economic incentives: most of the target population cannot afford expensive medication. As a consequence, such development projects invariably carry a huge financial risk, and pharmaceutical companies have been reluctant to initiate drug-development projects against organisms and target proteins with which they have had no previous experience.

- We propose a novel approach based on selecting, as potential drug targets, enzymes that are strongly conserved between the human host and the parasite. If such a human enzyme is already explored as a drug target, and if the corresponding parasite enzyme is essential for survival of the parasite, available expertise and technology can be applied to develop inhibitors that are fatal for the parasite. This will greatly facilitate the development of parasite-specific drugs and, thus, will considerably diminish the financial risk of such projects.

- The concept is exemplified here by using cyclic nucleotide-specific phosphodiesterases (PDEs) as an example. Crystallographic analysis has demonstrated that these enzymes are highly conserved between humans and the parasites Trypanosoma brucei or Leishmania major.

- Biological experiments in vitro and in vivo have demonstrated that such PDEs are essential for parasite survival and for maintaining an infection.

- High-throughput screening of a proprietary compound library (Nycomed Pharma) with recombinant PDE from T. brucei using the company's standard operating procedures has revealed a number of highly potent compounds.

- Crystallographic analysis of the parasite PDEs has provided information on subtle structural differences between the human and the parasite PDEs that can now be exploited to synthesize optimized lead compounds. 
Key Terms

Kinetoplastida: Systematic order of flagellated, unicellular eukaryotes. Most members of this order are parasites of multicellular organisms, and several are medically and economically important disease agents of humans, their domestic animals and their cash crop. The medically important species are transmitted to humans via insect vectors, including the tsetse fly (Glossina ssp; Trypanosoma brucei), the sandfly (Phlebotomus ssp or Lutzomya ssp and the various Leishmania) or bedbugs (e.g., Rhodnius prolixus and Trypanosoma cruzi).

Sleeping sickness: Inexorably fatal infection of the central nervous system caused by the kinetoplastid protozoa, Trypanosoma brucei gambiense and Trypanosoma brucei rhodesiense. The disease is endemic in 38 countries of Sub-Saharan Africa, with an estimated incidence of approximately 50,000 cases annually. It is a typical neglected disease that affects the inaccessible, poor rural areas of the countries. Together with the infections of domestic animals caused by the same and related trypanosomes, sleeping sickness constitutes a major burden on the afflicted populations and a major impediment to the economic development of these areas.

Leishmaniases: Complex of several diseases that are all caused by closely related kinetoplastid protozoa, the Leishmania ssp. The protozoa are transmitted by bloodsucking sandflies. Depending on the clinical course and the disease agent, three major disease forms are distinguished: cutaneous leishmaniasis (usually self-healing, but leaving ugly scars), visceral leishmaniasis (frequently fatal) and mucocutaneous leishmaniasis (frequently fatal).

Protozoa: Diverse group of single-cell eukaryotic organisms, including many pathogens. In contrast to bacteria, the protozoa are genetically and biochemically much more closely related to higher organisms, including mammals. This fact traditionally made the development of selective chemotherapy much more difficult.

RNA interference: Molecular technique that allows the elimination of a specific target mRNA from a living cell. The technique allows the quick exploration of whether a given protein is essential for the target cell. Interfering RNA can be synthesized chemically and introduced into the target cells from outside (e.g., via electroporation), or it can be genetically encoded by a DNA construct that is stably integrated into the target cell genome. The latter mode allows selective induction of RNA interference by placing its expression under the control of an inducible repressor. 


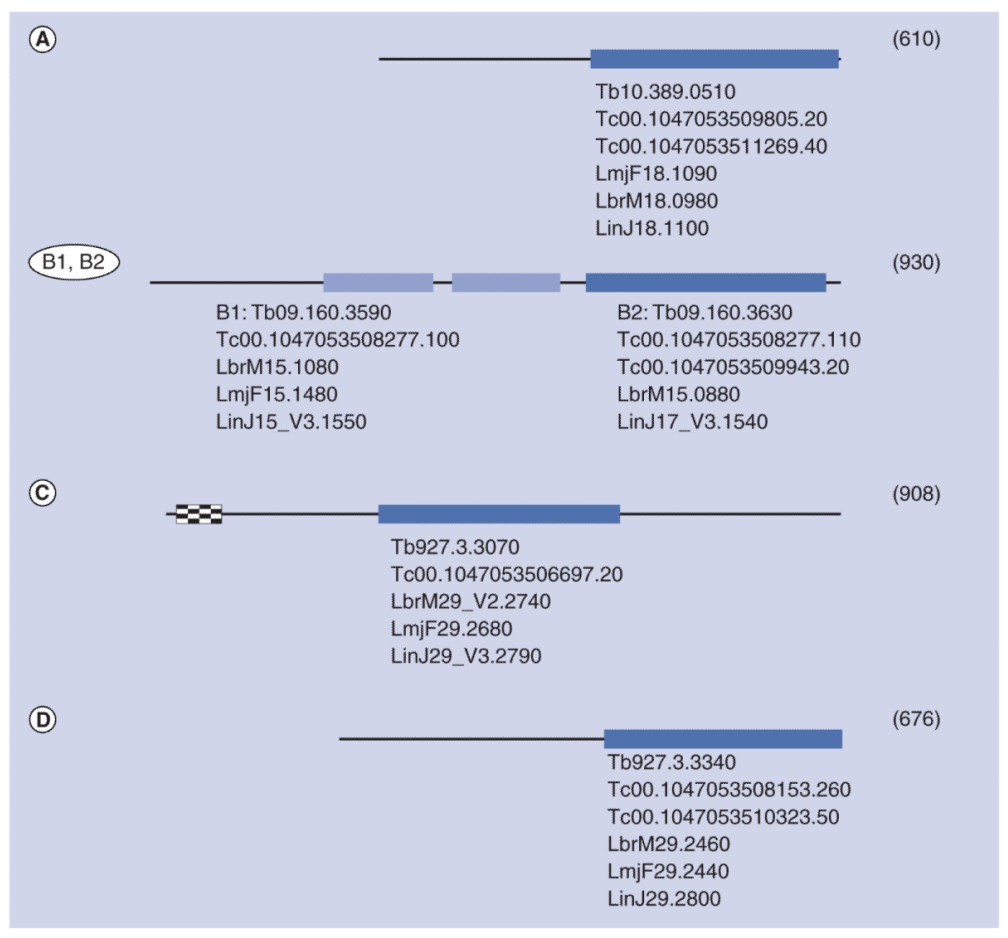

Figure 1. Schematic representation of phosphodiesterases A-D encoded by kinetoplastid genomes

Darker boxes: class 1 catalytic domains; lighter boxes: GAF domains; checkered box: FYVE domain. Size in amino acids of each enzyme (of T. brucei) is indicated in parentheses. Gene identification numbers [208] are given underneath each scheme. Lbr: Leishmania braziliensis; Lin: Leishmania infantum; Lmj: Leishmania major; Tb: Trypanosoma brucei; Tc: Trypanosoma cruzi. 


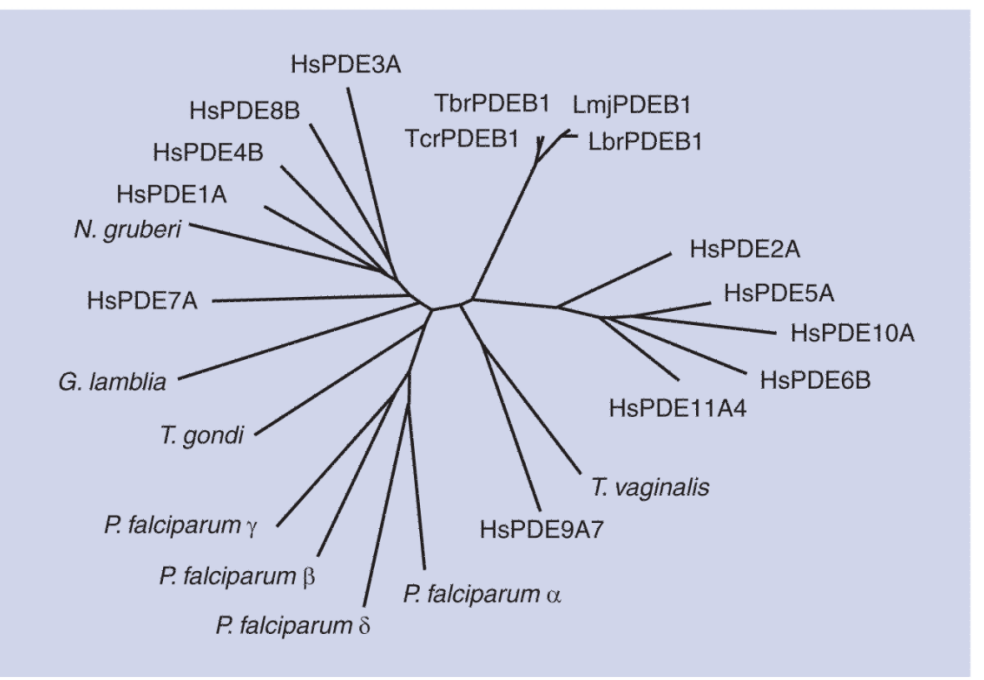

Figure 2. Unrooted tree of catalytic domains of the phosphodiesterases of human and protozoan origin

The following sequences were used for ana lysis: HsPDE1A, accession number P54750;

HsPDE2A, O00408; HsPDE3A, Q14432; HsPDE4B, Q07343; HsPDE5A, O76074; HsPDE6B, P35913; HsPDE7A, Q13946; HsPDE8B, O95263; HsPDE9A7, Q86SI6; HsPDE10A, Q6FHX1; HsPDE1A4, Q9HCR9; TbrPDEB1 (Trypanosoma brucei), AAK33016; LmjPDEB1 (Leishmania major), AAR88146; LbrPDEB1 (Leishmania braziliensis), A4H8B6; TcrPDEB1 (Trypanosoma cruzi), XP_820270; PfPDEa (Plasmodium falciparum), ABS50256; PfPDEb, ABS50258; PfPDEg, ABS50259; PfPDEd, ABS50260; GIPDE1 (Giardia lamblia), Q7QRM3; TvPDE1 (Trichomonas vaginalis), A2E0E8; NgPDE1 (Naegleria gruberi), XP_002682684; TgPDE1 (Toxoplasma gondii), B9PN68. Sequences were aligned using the MAFFT algorithm. 


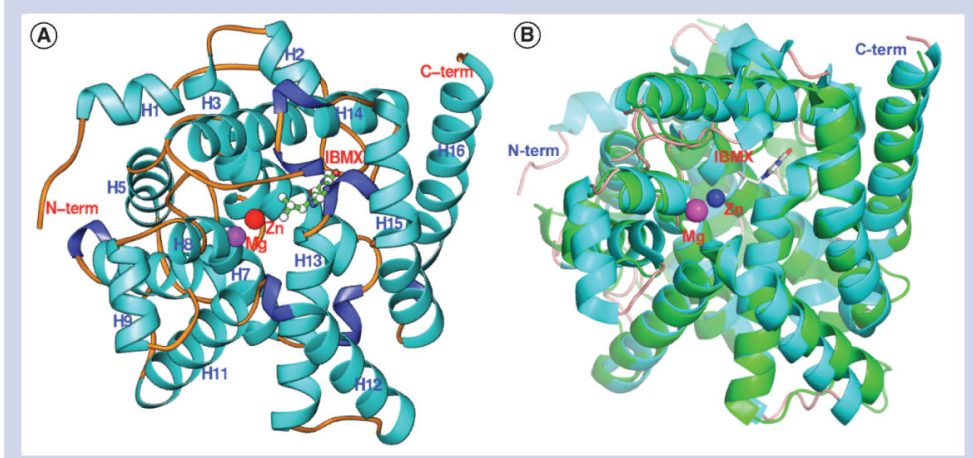

Figure 3. Structure of LmjPDEB1

(A) Ribbon diagram of the LmjPDEB1 catalytic domain. (B) superposition of LmjPDEB1 (cyan) over human PDE4D (green). All structural figures were generated using the Ribbons software package [93]. 


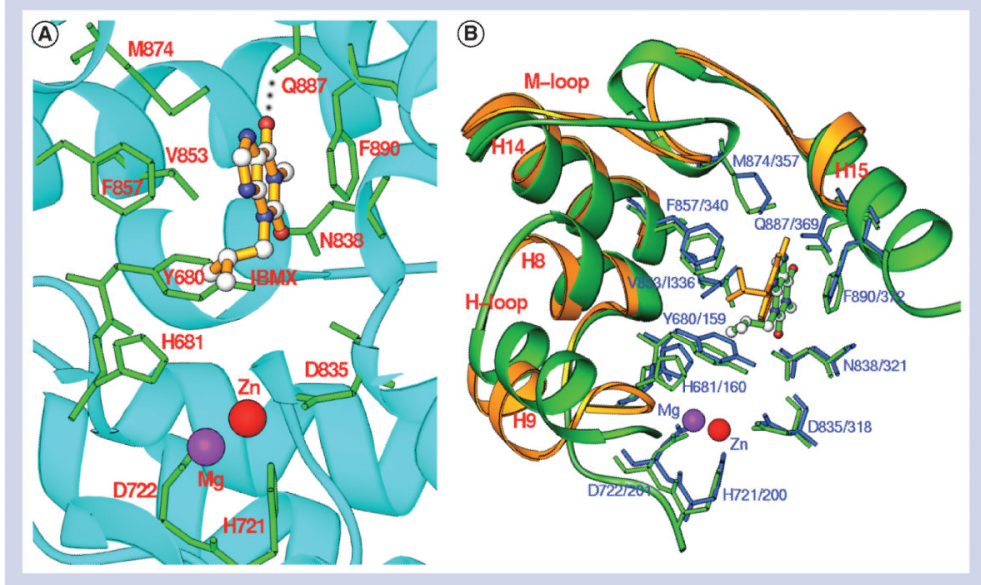

Figure 4. 3-Isobutyl-1-methylxanthine binding

(A) Interaction of 3-isobutyl-1-methylxanthine with the LmjPDEB1 active site pocket. (B) Superposition of LmjPDEB1 (green) over human PDE4D (gold and blue) interacting with 3isobutyl-1-methylxanthine. 


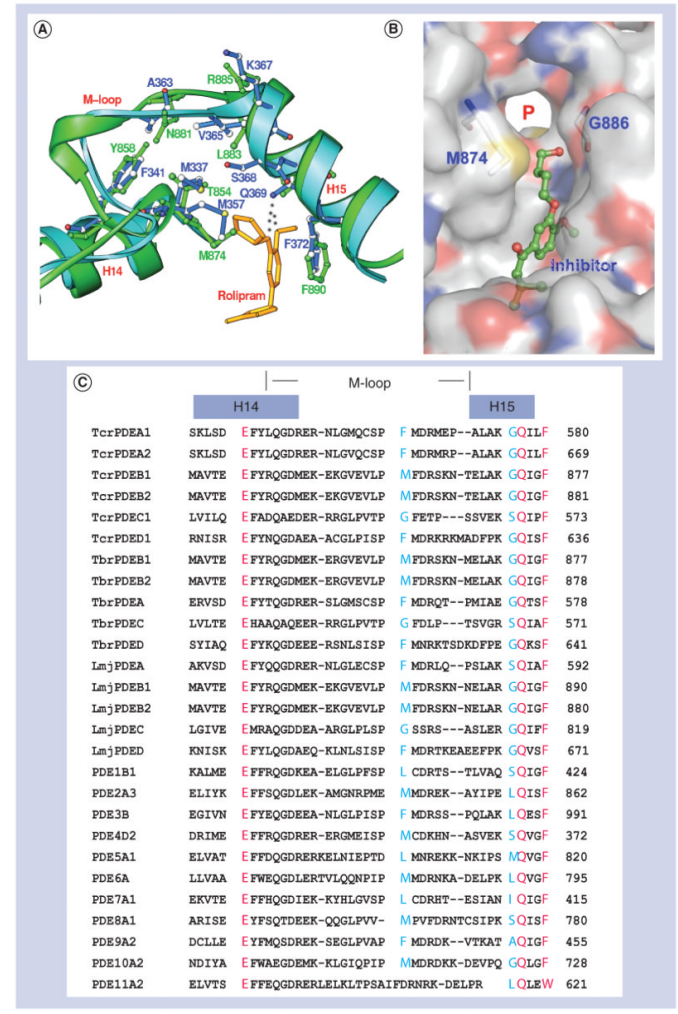

Figure 5. The putative parasite pocket (P-pocket)

(A) Superposition of the M-loop region of LmjPDEB1 (green) and of human PDE4D (blue). The positioning of Rolipram is taken from the co-crystal of human PDE4D with rolipram. (B) Surface presentation of the parasite pocket (P-pocket) in LmjPDEB1. (C) The sequence alignment of the pocket among trypanosome, Leishmania and human PDEs. The red color marks the identical amino acids in all phosphodiesterases. Two blue residues (Met-874 and Gly-886 in LmjPDEB1) play a gatekeeper role to isolate the parasite pocket from the active sites of human phosphodiesterase families [71]. 


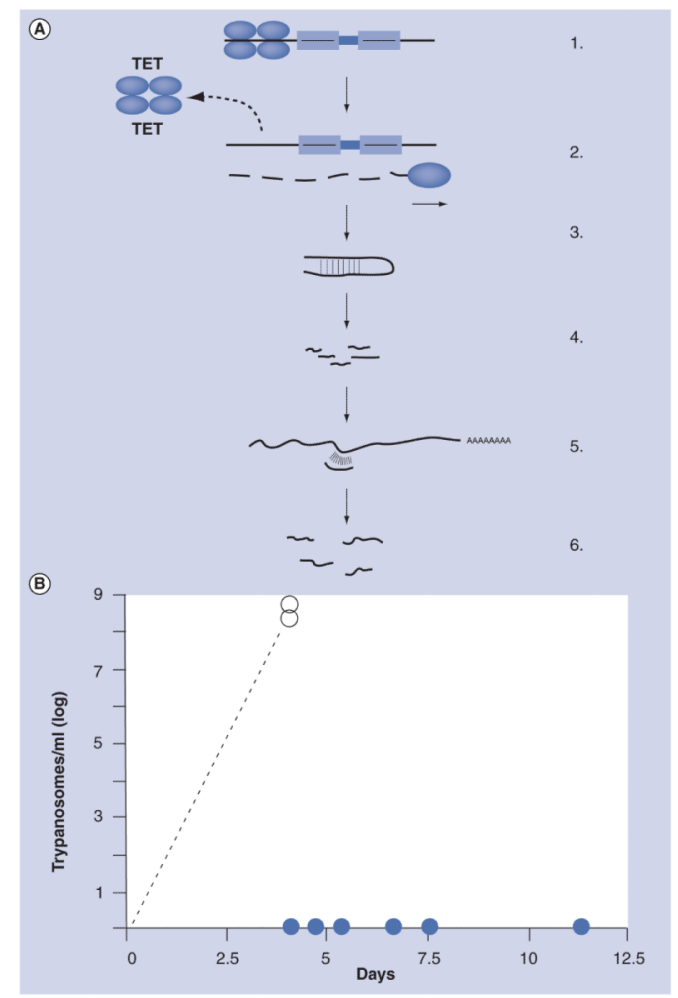

Figure 6. TbrPDEB1 and TbrPDEB2 are essential for trypanosome virulence

(A) Principle of tetracycline-inducible RNA interference (RNAi) [94]. 1: Genetic construct containing a partial sequence (a few hundred base pairs) of the target mRNA, in opposite orientations (arrows). In the absence of tetracycline, transcription is prevented by the upstream binding of a tetracycline repressor; 2 : In the presence of tetracycline, the repressor dissociates and the sequence is transcribed; 3 : The transcript folds into a partially doublestranded hairpin structure; 4: This structure is recognized by the cellular RNAi machinery and is degraded into 23 nucleotide fragments; 5: These fragments bind specifically to the target mRNA to form partially double-stranded regions; 6: These are recognized by the RNAi machinery, and the target mRNA is completely degraded. (B) Effect of in vivo RNAi on the infection of mice. In animals that received tetracycline two days before the infection in the drinking water, RNAi is induced and the mRNAs for TbrPDEB1 and TbrPDEB2 are degraded. As a consequence, the parasites are killed, and no infection is produced (closed circles). Control animals (no tetracycline) acquire fatal parasitemias of $>10^{8}$ trypanosomes/ $\mathrm{ml}$ blood within 3-4 days (open circles). 


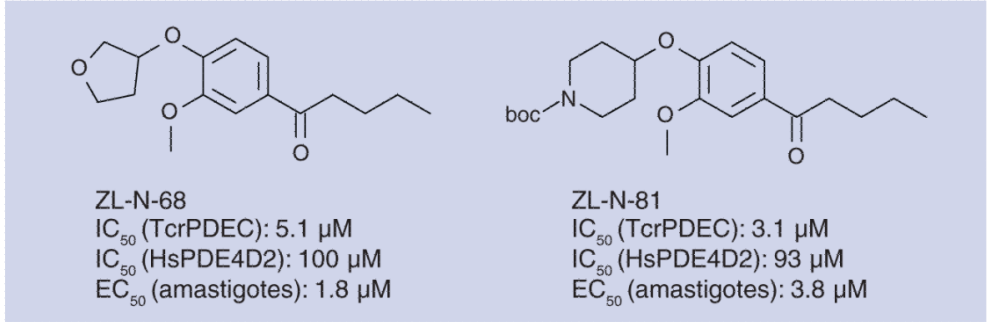

Figure 7. Catechol ethers with high potency towards phosphodiesterase C of Trypanosoma cruzi 


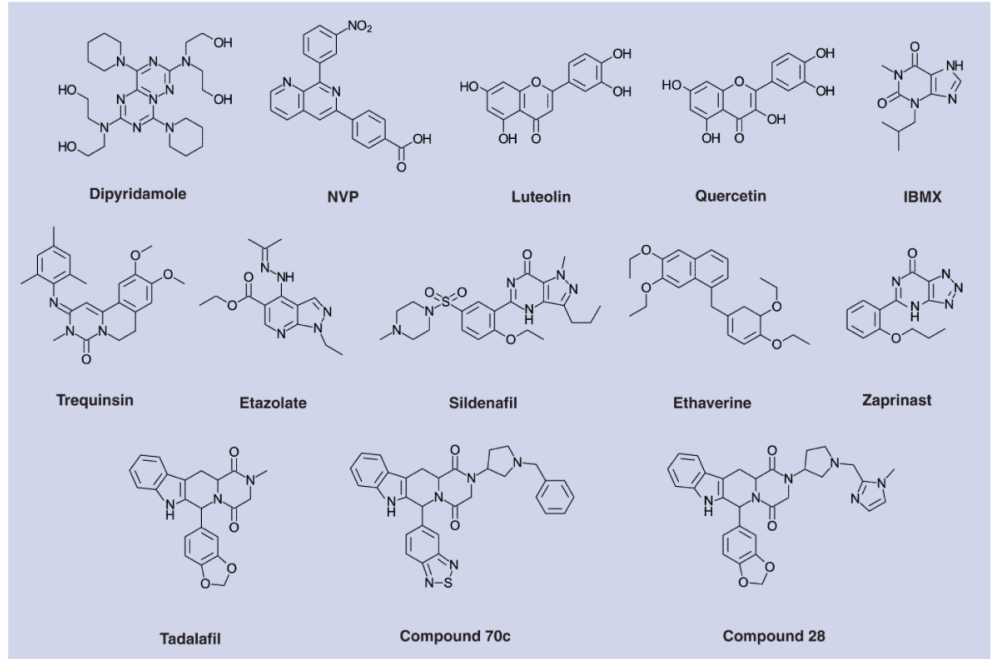

Figure 8. Phosphodiesterase inhibitors with demonstrated activity against trypanosomatid phosphodiesterases 


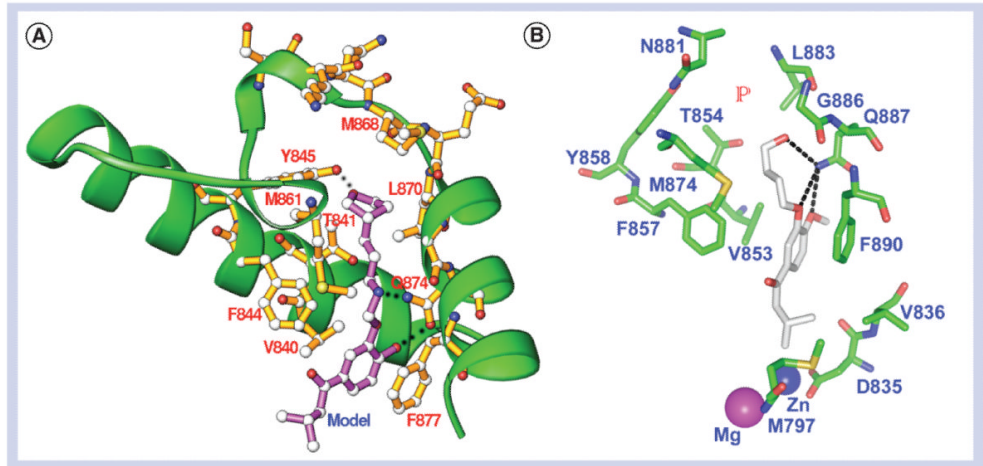

Figure 9. An inhibitor of LmjPDEB1

(A) A model targeting the P-pocket. (B) The compound 1-(3-(4-hydroxybutoxy)-4methoxyphenyl)-3-methylbutan-1-one binds to the active site of LmjPDEB1 and extends into the P-pocket. 
Table 1

Potency of selected phosphodiesterase inhibitors against TbrPDEB

\begin{tabular}{|ll|}
\hline Compound & $\mathbf{I C}_{\mathbf{5 0}}(\boldsymbol{\mu M})$ \\
\hline 3-Isobutyl-1-methylxanthine & 1704 \\
\hline Sildenafil & 42 \\
\hline Dipyridamole & 14 \\
\hline Etazolate & 36 \\
\hline Ethaverine & 27 \\
\hline Trequinsin & 13 \\
\hline
\end{tabular}

